\title{
Blog Management System Based on JSP
}

\author{
C.P. HAN \\ Science and Technology Institute of Shanxi Datong University, China
}

\begin{abstract}
Posting personal logs and interesting content has gradually become a new kind of way to show self and discover talented people in conternporary society, so blog was born. Servlet gives a great help to programmers. It can hardle the HTTP requeats from clients and reterns a response. Servlet is a Java-language class, it can basically realize the most functions that the Java-language can realize.This system will use many function modules such as "my article" and "photo album upload" to attract more visitors, increase site site traffic, improve the influential of the webcites; and continually update the site information content. Then the website information is more diversified. It is suitable for different groups of audiences.
\end{abstract}

KEYWORD: blog management; modular design; database

\section{INTRODUCTION}

\subsection{Based on the JSP blog management system} development background and design target

Along with the network popularization and the development of Internet technology, the communication channel is more wide, the blog also arises at the historic moment. There are a lot of bloggers on the Internet, but often slow access speed, the article can't release, server busy. Development of the personal blog website platform based on JSP. Users of this platform can be convenient to release information, writing personal journal, etc., in this platform we can communicate knowledge of JSP, and enhance their understanding of JSP technology. Blog management system based on JSP through "my article" and "photo upload" function module, such as to attract more visitors,Main application Servlet technology development blog system. Servlet technology brings is the biggest help programmers it handles HTTP request from the client, and returns a response. The Servlet is a Java class, the Java language can realize the function, the Servlet basically can achieve.

\subsection{Paper work and structure arrangement}

(1) the blog network system requirements analysis

(2) the blog network system architecture design

(3)the blog network system design and development of each module, ect.
This article is divided into five chapters, the structure of the thesis are arranged as following:

Chapter 1 is the introduction, introduced the topic background, the development goal.

Chapter 2 is the introduction of relevant technology, introduces the key technology needed to build a blog network system based on JSP.

Chapter 3 is the overall design, the overall framework of system design and the overall design of the database table structure.

Chapter 4 is the module of detailed design, the various modules of the system design in detail.

The fifth chapter is the conclusion, summarizes the design and implementation process of this system is analyzed.

\section{RELEVANT TECHNICAL INTRODUCTION}

\subsection{JSP profile}

The JSP (Java Server Pages) is advocated by Sun Microsystems company, many companies participate in together to establish a dynamic web technology standards. JSP technology is similar to the ASP technology, it is in a traditional web page HTML files (scriptlets) and insert the Java program in JSP tags (tag), thus forming a JSP file (*. JSP) .

JSP technology using Java programming language class XML tags and scriptlets, processing logic to encapsulate generate dynamic web pages. Page also can be accessed through the tags and scriptlets exist in the server-side resource application 
logic. JSP page logic and Web page design and display of separation, support reusable componentbased design, make development of a web-based application quickly and easily.

Web server in case of access to the JSP page request, the first to perform the procedures section, and then the results together with the HTML code in the JSP file returned to the customer. Insert the Java program can operate on the database, redirect page, etc., to achieve the function needed to build dynamic web pages. JSP and Java Servlet, is to execute on the server, usually to return to the client is an HTML text, so the client with the browser can browse.

The JSP page by the HTML code and embed the Java code. Server after the page is a client request to deal with these Java code, then the generated HTML page returned to the client browser. Java Servlet is JSP technology base, and a large Web application development requires Java Servlet and JSP to cooperate to complete. JSP with Java technology is simple and easy to use, fully object-oriented, with platform neutrality and safe and reliable, largely to the Internet all the characteristics of.

\subsection{The Servlet profile and its advantages}

The Servlet is generally expanded to support Java server. It is most commonly used to expand the web server, provide very secure, portable, easy to use cgi alternatives. It is a kind of dynamic loading module, provide service for a request from the web server. It is completely run in Java virtual machine. Because it to run on the server, so it doesn't depend on the browser compatibility.

This system mainly applies the Servlet technology development. Servlet technology brings is the biggest help programmers it handles HTTP request from the client, and returns a response.

\subsection{Introduction of the JavaBean}

JavaBean is a JAVA language reusable components. For written JavaBean classes must be specific and the public, and has a parameterless constructor. JavaBean by providing with consistency of design patterns public methods expose internal domain known as attributes. As is known to all, the attribute name conforms to this pattern, other Java classes can be found through introspection mechanism and operating the JavaBean property.

\subsection{Profile and its characteristics of SQL Server}

Microsoft SQL Server is a high-performance, client/Server RDBMS (relational database management system), to be able to support the large volume of transaction processing, as well as Microsoft Windows Server 2000.
Manage data access under the network environment and the development of decision support applications. Due to the Microsoft SQL Server is an open system, other systems (e.g., based on the UNIX system) can interact with it in good condition of operation [4]. One of the most representative is SQL Server 2000, its characteristic is as follows:

(1) the real client/server architecture.

(2) the graphical user interface, make the system more intuitive, simple management and database management.

(3)rich programming interface tools, programming for users provide greater choice.

(4) the SQL Server with Windows NT fully integrated, using many of the features of NT, such as send and receive messages, manage login security, etc. SQL Server can also be well integrated with Microsoft BackOffice products.

(5) has a good scalability and can span from running Windows $95 / 98$ of the laptop to run Windows 2000 large multiprocessor and other platforms to use.ect.

\section{OVERALL DESIGN BLOG MANAGEMENT SYSTEM BASED ON JSP}

\subsection{The system design}

\subsubsection{The system function structure}

The blog site is divided into foreground and background. Among them, according to the characteristics of the blog at the front desk, it can be divided into blogger information, network calendar, this paper queries and album query 13 sections.

According to the characteristics of the blog network background, it can be divided into user Settings, public announcement management, personal photo album management, post management, information management, information management and voting blogger modify part 7 .

\subsection{2 development environment}

In the development of blog network system, needs to have the following software environment.

The server side:

Operating system: Windows xp

The Web server: Tomcat 6.0

The Java development kit: JDK 1.5 above

Database: SQL Server 2000

\subsection{Database design}

\subsubsection{Database needs analysis}

Create blog database of the first step is to develop a plan of implementation, the plan can be implemented in the database to do the wizard, can also be realized in the database, as the function of 
the database. The complexity of the database design and details by the database application complexity, size, and the user usage sure [5]. Introduced here in the blog database is a relatively simple database.

Detailed below to database design basic steps:

(1) collecting information

Before creating the blog database, must have a thorough understanding of blog information. And everyone involved in the blog network to communicate, to find out what they are doing and they need from the database content, it is very important. In addition, to understand the blog system conformed to the actual requirements and find out the related systems, restrictions, and the problem of shielding is also very important.

(2) identify objects

In the process of gathering information, must be identified by the blog database management of the main object or entity. Objects can be a tangible entity (such as user), or it can be a invisible project (articles, album). Several major object, usually after identifies the object, the relevant project will become clear. The database every different project should have a corresponding table.

(3) establish object entities

Identifies the objects in the blog system, using a way of visual representation of the blog system to record these objects is very important. During the database implementation, you can use the database entity as a reference.

(4) identifies each information type of objects

Logo have to be stored for each object types of information, the information is the object in the table column (in fact is the type of database field).

(5) identify the database one advantage is that it has a contact or associated information about various items in the database. Can be separately stored independent information types, but the database can be combined data when necessary. In the design process, identify objects need to look at the relationship between all kinds of table, to make sure they are logically related way and add the relational columns to set up a table with another table relationship between.

\subsubsection{Database conceptual design}

According to the above on the system requirement analysis and system design, planning out the database entities are used in the system user information entities, announcements, information entities, personal photo album information entities, friends entity, voting information entities, blog information entities, and the article entity. The following will introduce a few key entities e-r diagram.

(1) the user information entities.

User information entities including username, password, name, sex, QQ number, home page, interest, E-mail addresses, property management level.

(2) personal album information entities.

Personal photo album information entities including photo albums storing the server address, photo album description and photo albums to upload time attributes.

\section{THE MODULE DETAILED DESIGN}

\subsection{The module design}

In the development process, often used some public classes, such as database connection and operation and string processing, therefore, in the development system, first should write these public class. What we need in the following will introduce the specific blog network public class writing process.

\subsubsection{The preparation of the database connection and operation}

This website USES SQL Server 2000 database system. Installed SQL Server 2000 JDBC Driver you can write with the database connection class. JDBConnection. Java provides not only the database connection, and, according to a Statement and a ResultSet database access com. Wy. Tool. JDBConnection class encapsulates the operations on the database.

\subsubsection{The preparation of the string class}

In the use of JSP development site often want to filter some special characters or convert some characters, these features are usually need to write a separate class to complete.To consider when the output of the JSP Chinese Chinese garbled question, it is also a very headache thing to some JSP novice. Different JSP application server corresponding to different JDK version, the method to solve the problem of garbage is not the same. This example is to use the String class getBytes () method to solve this problem.This website is to use servlets and JavaBean combination of technology development. JavaBean technology implementation to the operation of the database does not need any configuration in an XML file, and the normal operation of the Servlet program also need proper configuration, the configuration file for the web.

\subsection{The front desk page design}

\subsubsection{The front desk page overview}

The blog network, in the design of the front page of the first administrator to release the latest articles to show to the user, and then provides the classification of the article, according to the blogger information, the latest announcement shows and blog network navigation area function, and other functions. 


\subsubsection{The front desk page technical analysis}

On the front page of the display the basic information of the blogger, login user information, and display the main functions of the blog, blogger, circle of friends and copyright information, and other functions, is not only with the home page, in the other functional module of child pages also need to include these parts. Therefore, these parts can be separately stored in a separate file, when these functions need to be placed just contains these files.

\subsection{User login module design}

\subsubsection{Description of the user login module}

The user login module has the user login function. Blog network operation is the first page of the user login page, user login, you can view articles published by bloggers. In user login page, in the "user name" and "password" in the text box input user name and password, click "login" button, after the success of the system verification, users will be to the identity of the members into the blog network home page, then on the site are free to view the article released blogger

Chapter. Himself, if the login user is blogger in blogger information display area, is a hyperlink, "into the background" blogger can directly click the hyperlink, into a web blog background.

\subsubsection{The user login module technical analysis}

Database is involved in the user login module, user information table, preserved in the user information such as user name and password information, according to the information to create the from user login module, name ConsumerFrom.

The Servlet is at the heart of its HttpServlet, generally in the Servlet do the logical jump page. User login module Servlet implementation class inherits the HttpServlet class, first in the class set the user login module ConsumerDao class object, the object instantiated in each concrete method, doGet () in the Servlet is invoked automatically. The method itself has no specific transaction, it is based on a through it the getParameter () to obtain the method of parameter value to perform the corresponding method.

\subsubsection{User login module realization process}

User login module is the blog network function of the first to use, is the entrance to the system. In the user login page, users can input the correct user name and password to enter into the system, when the user didn't enter the user name and password, the system can judge by JavaScript, and give prompt information.
(1) design the user login page

The user login page is mainly used to collect user input information and pass the custom JavaScript function to determine whether a input information is empty.

(2) write a user login Servlet implementation class

In the user login to the "user name" and "password" in the text box input the correct user name and password,

Click "login" button, to pay a visit to a web URL, namely ConsumerServlet? Method $=0 \&$ sign $=0$.

Can know from the URL in the address user login module involves the method of parameter value is 0 .

(3) write the user login ConsumerDao class methods

We know from the above introduction, the user login using ConsumerDao class method is getConsumerForm (). In getConsumerForm () method, with the user name information form for the parameter, to the user information in a table user named condition, perform the SQL query, and return the query results through the return keyword, if there is no query results, it returns null objects.

(4) pages according to the results of the validation Whether user validation result is successful, the implementation of user authentication Servlet will eventually return to dealwith. The JSP page, according to the ConsumerServlet? Method $=0 \&$ sign this URL $=0$, can know when sign $=0$, will perform according to the result of the user authentication code.

\section{REFERENCES}

[1] Guo-ping xu. 2001. The JSP web development practices. Electronic industry press: 156-170.

[2] GengXiangYi yue-ping zhang. 2003. JSP practical tutorial. Tsinghua university press, 101-120.

[3] Ed Roman, John wiley\&Sins. 2002. Mastering Enterprise JavaBeans Second Edition Inc.

[4] China Machine Press: 60-62.

[5] Chen Lian cheng, Chen. 2007. SQL Server 2000 practical tutorial. Second edition, Beijing: electronic industry press: 100-150.

[6] Zhang Haifan. 2007. Introduction to software engineering. 4th edition. Beijing: Tsinghua University press: 101-126.

[7] Ding Baokang Dong Jian 2006.compiled. Database practical tutorial. Second edition. Beijing: Tsinghua University press: $371-374$.

[8] xian-guo wang. 2006. JSP dynamic web programming techniques. Electronic industry press: 98-120.

[9] cheau-jane peng, li-wen yao. 2004. The online course selection system based on JSP technology. The design and implementation of computer and modern 4 (8): 123-124.

[10]Ek (Eckel, b.) ping, Chen wu et al. 2005 the JSP programming thought. 3rd edition. Beijing: mechanical industry press: 200-230. 